DOI: 10.2478/ausfm-2014-0013

\title{
Visuality and Narration in Monsters, Inc.
}

\author{
Jens Schröter \\ University of Siegen (Germany) \\ E-mail: schroeter@medienwissenschaft.uni-siegen.de
}

\begin{abstract}
The overblown rhetoric concerning the "digital revolution" conceals deep continuities between traditional and new forms. As the example Monsters, Inc. shows established forms of narration can be used together with new forms of computer generated images. The complexities of this constellation are described by an analysis of the film.
\end{abstract}

Keywords: digital revolution, narration, computer graphics, neoformalism, Pixar.

When talk turns to changes in media constellations, one of the transformations most discussed in recent decades must surely be the omnipresence of digital media. This change has often been accompanied by overblown rhetoric suggesting a profound break with the past. There were voices predicting, for example, that digital images would lose all relation to the world, and that virtual reality would become indistinguishable from the world, or, at least, that completely new, interactive, hypertextual, etc. aesthetic forms would emerge. And yet it has become more apparent over the years that perhaps not everything is changing and that perhaps many cherished aesthetic forms of composition and narration are still with us. We can still distinguish images which are intended to make reference to the world from those which do not do so, or not directly; in other words, fiction is still basically distinguishable from reality and many of the established narrative conventions are still in use.

Instead the question to be asked is what forms have been preserved or changed, in what contexts, and in what way. We need to switch from global theses to more detailed analyses illustrating continuities and discontinuities in individual cases. I would like to demonstrate this with the film Monsters, Inc. from Pixar, 2001. This seems to me to be well-suited as an example: firstly, it is one of those completely computer-generated films which thus stands paradigmatically for the shift to a digital media culture. In 1995, Pixar had produced the first of these films, 
Toy Story, to considerable acclaim. Secondly, however, its relative intelligibility it is addressed to children, after all - shows, prior to any theorizing, that there are evidently no radically new patterns of narration or composition in use here, these normally result in a deliberate reduction in intelligibility. The film thus seems to combine discontinuity with continuity. To investigate this, I will proceed as follows: In the first section I would like to make a few preliminary remarks about theory and method, to form the basis for the following analyses. This leads to the $2^{\text {nd }}$ section, in which I take a look at the narrative structure of Monsters, Inc., and to the $3^{\text {rd }}$ section, which is concerned with the visual imagery of the film, and with whether and how this relates to the narrative structure. This brings me, finally, to the $4^{\text {th }}$ section, in which I discuss the highly self-reflective nature of Monsters, Inc., something which seems to me to be far from coincidental. It seems as though the film not only stands at the threshold between traditional and new forms, but also that it draws attention to this historical situation itself.

\section{Transmedial and Transmaterial Forms}

The thesis that the digital "new media" mark the start of a radical revolution which, at the very least, will turn media culture inside out, is problematic because, for one thing, it assumes that forms appearing in media can only arise from the specifics of their media. Only if this were the case would new media more or less automatically bring forth completely new forms. This, however, overlooks the fact that forms can also be transmedial (cf. Schröter 2011).

This means that there are forms which appear in identifiable guise in artefacts of varying media provenance. A relatively simple example is central perspective, which was developed around 1425 and codified for the first time (in mathematical terms as well) in Alberti's De Pictura in 1435. It is an optional procedure for the representation of a pictorial space, which is available to painting, but does not necessarily have to be followed (other cultures have favoured other procedures, e.g. parallel perspective, see below). In technical visual media which follow geometric optics, such as photography, film, or video, this mode of representation must be followed (borderline cases occur when certain kinds of telephoto lenses are used). In digitally generated images, on the other hand, central perspective is optional, since, as Friedrich Kittler $(2001,35)$ once put it, "computer graphics make optic modes optional at all." Central perspective is a mode of composition found in images across different media, and can be formally identified in a comparison of images by means of the diagonal vanishing lines, which lead to a vanishing point. In the 
history of computer graphics, incidentally, the computer scientists who developed algorithms for representation using central perspective are known to have studied the relevant textbooks from the Renaissance (and later) and the instructions given there - some of which were already formulated in mathematical terms. This also shows that new media do not simply adopt older forms in a transitional phase - as is sometimes assumed - in order to get close to the audience. That may certainly play a part to begin with, but why should one forego established forms later on? Would it not be nonsensical to artificially restrict one's own creative options? And is this not even more the case with digital technologies which by definition, due to their programmability, have few specific forms of their own?

Another important transmedia form that can be used by very different media is narrative structuring of audiovisual media in time. Thus for example the narratologist Seymour Chatman (1981, 117, emphasis mine) once noted: "One of the most important observations to come out of narratology is that narrative itself is a deep structure quite independent of its medium." Admittedly this thesis has repeatedly been subjected to critical discussion, but it does seem to have some validity at least: if it were not so, there would be no film adaptations of literature. In section 2) I will outline the transmedia structure of the narrative in Monsters, Inc. Neoformalism seems a suitable theoretical framework for this; Bordwell (1993, 51), writes, for example: "As a distinction the fabula/syuzhet pair cuts across the media. At a gross level, the same fabula could be inferred from a novel, a film, a painting, or a play." Fabula is his expression for story, syuzhet his expression for the plot (more or less, in any case).

This does not mean, however, that all forms are transmedial, and equally available to all media. Painting and drawing have always also included modes of representation using parallel perspective, which have no vanishing point and which are still preferred in technical drawing and architectural drafting because they avoid changes in angle and relative changes in length (cf. Beil/ Schröter 2011). Photographic media cannot represent such forms (they can only approximate them in the borderline case of certain telephoto lenses), since they follow the behaviour of the light, whether their mode of recording is chemical, electronic, analogue, or digital. Computer-generated images, on the other hand, since they can represent anything which is computable within a reasonable time, can also use forms based on parallel perspective. This means that it is necessary to analyse precisely, in each specific case, which forms have been connected with which other forms and in what way - and to which media these forms are available or unavailable. 
There is, however, another point which must be considered when it comes to digitally generated images: insofar as such images are based on processes of computer simulation, they are not only able to pick up forms which are already transmedial anyway; they can also, partially and approximately, treat as form that element which has been considered, in the analogue media, as the other side of form, i.e. the materiality of the medium.

What is computer simulation? On the basis of collected or sampled data of various kinds it is possible to derive rules for or at least regularities in the behaviour of an object or process, a theory ("base model"). The base model is then translated into a computer-executable formalized model ("lumped model"). This formalized model must then be validated by aligning it with experimental data. Such procedures have been and are used for climate models, for example, or for other scientific prognoses (see Raser 1972). Now it is also possible to simulate other technological media. There are many examples of this, e.g. computergraphic photorealism. Photorealism is simulation, because the qualities (particular features) of photographic media are measured and the computer models are based on these data. A simulated or virtual camera is a real camera which, in accordance with the available data, can be brought ever closer to its material prototype (if this is what is desired). This virtual camera is now used to take a virtual photograph of a virtual object field, which is lit by a virtual source of light. With regard to their visual appearance, images generated in this manner follow, insofar as this is desired and computable, the fundamental characteristics of chemical photography: firstly, the wealth of unintended details. Secondly, the effects caused by the camera optics must be mentioned. Computer-generated images could also obey other logics of projection, but if they are intended to be photorealistic they follow the linear or central perspective-based structure passed down through photography and film. Thirdly, the aim is to model the qualities of the photographic emulsion itself, e.g. the grainy structure of the image, particularly in enlargements or very light-sensitive films (cf. Schröter 2003).

Insofar as computer simulation can itself partially and approximately transform the materiality of analogue media into forms, I would speak here of transmaterial - in contradistinction to: transmedial - forms. Such forms are new, quite simply because the medium/form difference of the analogue media becomes a form itself in the medium of the digital. They are different from transmedial forms: transmedial forms point to no specific medium; transmaterial forms point to a media-specific materiality, albeit in a different medial context. So what is the situation with transmedial and transmaterial forms in Monsters, Inc., a computer-simulated film? 


\section{Transmedial Narrative in Monsters, Inc.}

If we first consider the transmedia structure of Monsters, Inc. on the level of the audiovisual narration, we can note - to preview the results - that the film follows the "classical Hollywood mode of narration" as described by Bordwell, Thompson et al. for Hollywood cinema from about 1917. This applies to the movements of the virtual camera, which could of course fly around at will: Craig Good (quoted in Siebert 2005, 182), responsible for the post-production of Toy Story, commented: "We wanted the audience to respond to traditional dolly and crane movements, not to make them dizzy." There has clearly been a process of transfer of established forms into the aesthetics of digital media. The narrative structure of Monsters, Inc. cannot be analysed in detail here. A few remarks must suffice.

Bordwell (1986, 18) writes: "The classical Hollywood film presents psychologically defined individuals who struggle to solve a clear-cut problem or to attain specific goals." Clearly this also applies to Monsters, Inc. There is no indistinct blurring of objectivity and subjectivity, as in many forms of what Bordwell (1993, chapter 10) refers to as the "art cinema mode of narration." Instead, a clear situation is established at the outset: Sulley and Mike work at the company Monsters, Inc., after which the film is named, and are depicted as successful and, in this sense, career-oriented monsters; a subtle rendering of their facial expressions shows a psychological inner life which, for example, clearly associates success with enjoyment. And then, with the accidental entry of the small child (Boo) into the monsters' world, a problem arises which upsets the stable situation. For the remainder of the film Sulley and Mike try to solve the problem, i.e. to return Boo to her world, facing various complications on the way. And in the end, they succeed. The whole construction of the film serves to build up the causal steps of this chain of action as clearly and unambiguously as possible. Bordwell (1986, 27, 28): "Most explicitly codified into rules is the system of classical continuity editing. The reliance upon an axis of action orients the spectator to the space" and: "Most Hollywood scenes begin with establishing shots, break the space into closer views linked by eyeline-matches.” This classic structure can be found in precisely this form in Monsters, Inc.

I will analyse one sequence. Before beginning their work at Monsters, Inc., Sulley, the furry monster and Mike, his round, green friend, get ready in a sort of changing room: [Fig. 1] $1^{\text {st }}$ shot: establishing shot, the space is established, along with a line of sight (eyeline match) between Mike and Sulley; [Fig. 2] $2^{\text {nd }}$ shot: the antagonist, Randall, is introduced, a new line of sight is created between him and 
Mike; Mike gets a fright and jumps over the bench to Sulley's side (incidentally, the psychological depiction of the characters can be studied particularly well by watching Mike's face here), the eyeline match remains in place, however; [Fig. $3-6] 3^{\text {rd }}$ to $6^{\text {th }}$ shots: a classic sequence of shot/reverse shot begins here, whereby the virtual camera always remains on this side of the eyeline, i.e. it observes the 180 degree rule; [Fig. 7] $7^{\text {th }}$ shot: there is another long shot which makes the spatial configuration absolutely clear again. In short: the construction of the space is completely focused on consistency. The space is intended to be the stable background for the development of the causal chains of action by the protagonists and antagonists, and is not supposed to confuse matters by intervening itself. This is typical of the classic Hollywood film. Deviations from this, such as a conspicuously tilted line of sight [Fig. 8], are only permissible because this is a still from a hectic chase situation, Bordwell $(1986,27)$ : "Stylistic disorientation, in short, is permissible when it conveys disorienting story situations."

In short: the film confirms the assertion that "classical narration quickly cues us to construct story logic (causality, parallelisms), time, and space in ways that make the events 'before the camera' our principal source of information" (Bordwell 1986, 24). But: in a computer-generated film there actually is no "before the camera" (unless we count the virtual space "in front" of the virtual camera, but that's quite metaphorically). It is significant that, during the closing credits of the film, (very amusing) "bloopers" are shown, constructing "pre-film events" with an ironic wink: the clapper board, a microphone in the picture, and finally an out-of-control machine which knocks over the "camera." Here Monsters, Inc. is of course ironizing its own mode of narration (and its "production culture," cf. Caldwell 2008) - in one of the "blooper" scenes a monster botches a dialogue, and is berated by his monster colleague: "You're messin' up this scene, we're never gonna work in Hollywood again.” Precisely: classical Hollywood narration. In short, Monsters, Inc., although completely digitally simulated, follows this classic narrative tradition.

\section{Transmaterial Visual Imagery in Monsters, Inc.}

The discontinuities must therefore lie on a different level. The obvious aspect is the visual imagery, the look of Monsters, Inc. [Fig. 9] is still organized using central perspective. Now computer graphics do not have to have central perspective, of course; unlike photographic media, the choice of central perspective in computer graphics is always a conscious stylistic decision, and here, of course, its purpose 
is to make the cartoon image seem photorealistic at the same time. The reference to the simulation of photography is obvious in many respects: for example when Sulley observes Randall pursuing his machinations from under a table, and the table legs and edges in the foreground are out of focus [Fig. 10]; or the other way around, when the background is out of focus [Fig. 11]. In photographic optics (be it photography, film, or analogue, or digital video) such varying levels of focus are part of the dispositif, in simulated images, on the other hand, they have to be wanted and brought about deliberately, e.g. in order to achieve a photorealistic effect. Fig. 11 also shows another typical way of getting closer to the visual imagery of photography, marking a considerable difference from many cartoon styles - that is, the numerous apparently random surface details. It is hardly necessary to point out that the photographic monocular is evoked even in the logo of Monsters, Inc., which is also one-eyed [Fig. 12]. This is taken to extremes - and here the meaning of the term transmateriality becomes particularly clear - when even faults in photographic optics are simulated, such as in Fig. 13, we see lens flares, which occur when shooting into the light with an optical lens system. But there is no material lens system in a simulated film. This effect is deliberately built in to reinforce the photographic appearance of the picture. (As an aside: there are programmes specially designed just to create such effects). So the point is: faults which result from the material specifics of media technologies behind the transmedial forms become transmaterial forms themselves. Here it is faults in photographic optics which are transferred into a completely different context, in this case the cartoon. For the visual imagery of Monsters, Inc. is not simply photorealistic: on the contrary, the film links photographic with cartoonish visual imagery, as can be seen in, amongst other things, the extreme colourfulness, especially of the shadows, see Fig. 12. This role of drawing and painting, the tradition to which cartoons and animation belong, is thematized intradiegetically at various points in the film, for example when the childish drawings produced by Boo point directly to the potential of non-photorealistic rendering (cf. Strothotte/Schlechtweg 2002, see Fig. 14). This hybrid form of image is the actual new visual/aesthetic achievement of the Pixar films (I exclude a few marginal predecessors in computer graphics research). ${ }^{1}$

1 Non-photorealistic rendering is especially interesting, since Kittler's (2001, 35) famous claim that "computer graphics make optic modes optional at all" does not cover drawn or painted pictures insofar they use conventions of representation (e.g. parallel perspective) that are not a form of optics - be it an optics describing the behavior of light (geometrical or wave optics), be it an optics describing the behavior of human sense perception (physiological optics). 
Above and beyond this - and the significance of this element should not be underestimated - a further aspect plays a part in the visual imagery of these films and thus also that of Monsters, Inc.. Pixar, the firm behind Monsters, Inc., was substantially built up with money from Steve Jobs, and does not only make money with films. Since 1989 it has also been selling software, PhotoRealisticRenderMan, based on the RenderMan standard. Pixar also defines the cutting edge of the computer graphics industry standard. ${ }^{2}$ Seen in this light, the films are also advertising for the graphic achievements of Pixar. The technical state of play definitely determines the choice of subject of the films. Hence Friedrich Kittler $(2001,36)$ noted in 1998 "Not coincidentally, computer generated films like Jurassic Park do not even attempt to compete with the fur coats [!] in Hans Holbein's The Ambassadors; they content themselves with armored and thus optically unadorned dinosaurs." But in Monsters, Inc. 2001 it was the rendering of fur and hair which was foregrounded, precisely because it had previously been difficult to simulate such complex structures convincingly. ${ }^{3}$ This is the reason for the narrative digression of Mike and Sulley's banishment to the Himalayas: when Sulley attempts to reach a nearby village he falls from the sled and lies in the snow, and his fur is blown about by the harsh wind and gradually covered by snowflakes. This scene demonstrates what was then the state of the art in the simulation of moving fur-like surfaces.

Knowledge of this function of the Pixar films can in itself become an attraction for viewers. Thus neo-formalist film theoretician Kristin Thompson commented: "For me, part of the fun of watching a Pixar's film is to try and figure out what technical challenge the filmmakers have set themselves this time. Every film pushes the limits of computer animation in one major area, so that the studio has been perpetually on the cutting edge." ${ }^{4}$ Certain elements of the film, then, are not simply subordinated to the narrative process. The lens flares, for example, have no function in the development of the causal chain, nor does Sulley's elaborate fur; furthermore this - in the words of David Bordwell - could at best be transtextually motivated, as something borrowed from a knowledge of the design of monster films. But they represent elements which can be understood in Kristin Thompson's terms as excess, or in Bordwell's terms as purely "artistically motivated” (Thompson 1986; Bordwell 1993, 36, 53, 164 and passim). These are

2 See: $h t t p: / / r e n d e r m a n . p i x a r . c o m / v i e w / r e n d e r m a n$ [last accessed: 22. 08. 2012].

3 See: http://renderman.pixar.com/products/whats_renderman/4.html [last accessed: 22. 08. 2012].

4 See: http://www.davidbordwell.net/blog/2006/10/08/reflections-on-cars/ [last accessed 22. 08. 2012]. 
elements which display their own fabricated nature and thus form a discourse about the state of development of the computer image, over and above the narrative. In this respect the new visual imagery of the Pixar films does in fact change the narrative. Although the narrative largely conforms to the "classical Hollywood mode of narration," it is - to use another term of Bordwell's (1993, 58, 59) - more "self-conscious" or "self-referential," since it does not merely conceal itself in order to seamlessly convey the story/fabula/information, as is usually the case in this mode of narration. Attention is increasingly focused on its own fabricated nature, to the point where one wonders whether the film's subject was chosen as a showcase for a specific new accomplishment of simulated visual imagery. The various processes of transfer, on the one hand of the form of photography into the digital image, and on the other hand of the form of classical narration into the arrangement of the digital images and sounds, therefore interfere with one another. That also means: different methods and theories have to be combined, here: media archaeology (Kittler) to explain the synthetic image and neo-formalist film theory (Bordwell, Thompson) to explain the narrative structure.

\section{On the Reflexivity of Monsters, Inc.}

The increased self-referentiality or self-consciousness of the narrative, which arises from its interference with the hybrid visual imagery (and the discourses surrounding this) in Pixar films and in particular in Monsters, Inc., reveals itself in the many self-reflexive references, some of which have already been mentioned. There are many more levels and ways in which the film is reflexive. Thus in Film Theory. An Introduction through the Senses Thomas Elsaesser and Malte Hagener $(2010,170-187)$ explicitly pointed out the role of the doors through which the monsters can enter the children's world in order to frighten them. This evokes discourses about the "portal to another world" which have, since the 1990s, referred directly to cyberspace and virtual reality (see Schröter 2004, 227). Furthermore, "Monsters, Inc." - i.e. the company our monsters work for - is an industry for the production of terror and (at the end of the film) laughter, so in this sense it is a reflection of the production of affect by the film industry. All that can be added to this precise analysis is that the motif of the door later expands into a massive archive of doors, a database; this in turn, to paraphrase Lev Manovich, introduces a new theme to the digital film: the logic of the database, which is typical of the new media (see Manovich 2001, 212). Furthermore, in the chase at the end of the film the doors function, as it were, as shortcuts through the diegetic 
space, which is at the same time global space, and allow a sort of montage within the image, which in turn displaces and reflects the forms of spatial construction in classical Hollywood cinema. The motif of the door would be worthy of a more detailed commentary.

I would like to finish, however, by discussing something much more straightforward. Monsters, Inc. begins in a simulator. The sequence is established with sounds off-camera, indicating that parents have put their child to bed; in the establishing shot (which is in fact the third shot) we see the child sleeping. The door - that portal to the monsters' world - opens. A monster has entered. It rears up to frighten the child, the child screams, and what happens? The monster gets the most dreadful fright itself, trips over a football, hurts itself: in short, messes everything up. Then the light goes on. A technical voice off-camera repeats again and again: "Simulation terminated," and we learn that the child was only a machine. And in a further doubling of the theme of the door to another world, one wall of the apparent child's bedroom slides up and we see the trainer as she tries to explain to the monster-in-training (and to the other monster trainees who are watching) what he has done wrong, in the first instance, this is an allusion to the diegetic $4^{\text {th }}$ wall. More important still: it is a simulator, just like those flight simulators which, in some respects at least, stood at the beginning of the development of certain forms of photorealistic computer graphics (see Schröter 2003). And one of the reasons why the simulator is established here is because it appears again later on. Sulley and Boo, on the run from the evil boss of the company - the classical evil capitalist of Hollywood cinema, later to be replaced by Sulley as the good capitalist - have apparently fled through a door into a child's bedroom. The evil boss, who is also behind Randall's machinations, wants to get hold of Boo, but when he reaches out to seize her from the bed it turns out that they are in the simulator. The evil boss is utterly confused. But that is not important any more, because he has just revealed his sinister plans to Sulley while in the simulator, thinking it was a child's bedroom. However, Mike was controlling the simulator, and has recorded the boss's crucial confession on a sort of video tape. This representation not only reflects back to another predigital visual form, in that the interlace lines are part of the simulation [Fig. 15]. More importantly, a turning point in the narrative is explicitly connected with the theme of simulation here. Here the interference between the narrative and the simulative visual imagery in Monsters, Inc. is itself thematized intradiegetically. 


\section{Very Brief Conclusion}

I would like to come to a very brief conclusion. My analysis has attempted to identify two different processes of transfer in Monsters, Inc. - in the narrative and in the visual imagery - which interfere with one another and thus represent a complex reaction to the changing media constellation as it shifts towards digital media. In Monsters, Inc. one can clearly see that media change does not - of course - lead to completely new forms, but that old and new processes and forms appear in new constellations. And this necessitates perhaps unexpected constellations of methods - e.g. combining media archaeology (Kittler), production studies (Caldwell) and neo-formalism (Bordwell). Pixar films seem a worthwhile object for an interdisciplinary dialogue about intermedial processes of transfer within the changing media. Is it a coincidence that a sequel to Monsters, Inc. came to the cinemas in 2013, with the title Monsters University?

\section{References}

Beil, Benjamin and Jens Schröter. 2011. Die Parallelperspektive im digitalen Bild [The Parallel Perspective in Digital Images]. Zeitschrift für Medienwissenschaft no. 4: 127-138.

Bordwell, David. 1986. Classical Hollywood Cinema. Narrational Principles and Procedures. In: Narrative, Apparatus, Ideology, ed. Phil Rosen, 17-34. New York: Columbia University Press.

Bordwell, David. 1993. Narration in the Fiction Film. London: Routledge.

Caldwell, John. 2008. Production Culture: Industrial Reflexivity and Critical Practice in Film and Television. Durham/NC: Duke University Press.

Chatman, Seymour. 1981. What Novels Can Do That Films Can’t (and Vice Versa).

In On Narrative, ed. W.J.T. Mitchell, 117-136. Chicago/London: University of Chicago Press.

Elsaesser, Thomas/Hagener, Malte. Film Theory. An Introduction Through the Senses. New York: Routledge.

Kittler, Friedrich. 2001. Computer Graphics. A Semi-Technical Introduction. Grey Room no. 2 (winter): 30-45.

Manovich, Lev. 2001. The Language of New Media. Cambridge, MA: MIT Press. Raser, John. 1972. Simulation and Society. An Exploration of Scientific Gaming.

Boston: Allyn and Bacon.

Schröter, Jens. 2003. Virtuelle Kamera. Zum Fortbestand fotografischer Medien 
in computergenerierten Bildern [Virtual Camera. About the Survival of Photographic Media in Computer Imagery]. Fotogeschichte vol. 23 no. 88: 3-16.

Schröter, Jens. 2004. Das Netz und die VirtuelleRealität.Zur Selbstprogrammierung der Gesellschaft durch die universelle Maschine [The Net and Virtual Reality. Society's Self-Programming through the Universal Machine]. Bielefeld: Transcript.

Schröter, Jens. 2011. Discourses and Models of Intermediality. In: CLCWeb: Comparative Literature and Culture. vol. 13, no. 3 (September). http://docs.lib.purdue.edu/clcweb/vol13/iss3/. Last accessed at 22. 08. 2012.

Siebert, Jan. 2005. Flexible Figuren. Medienreflexive Komik im Zeichentrickfilm [Flexible Figures. Media-reflexive Comic Effects in Cartoons]. Bielefeld: Transcript.

Strothotte, Thomas and Stefan Schlechtweg. 2002. Non-photorealistic Computer Graphics: Modeling, Rendering, and Animation. San Francisco, CA: Morgan Kaufmann.

Thompson, Kristin. 1986. The Concept of Cinematic Excess. In: Narrative, Apparatus, Ideology, ed. Phil Rosen, 130-142. New York: Columbia University Press.

\section{List of Figures}

\section{Figures 1-3.}
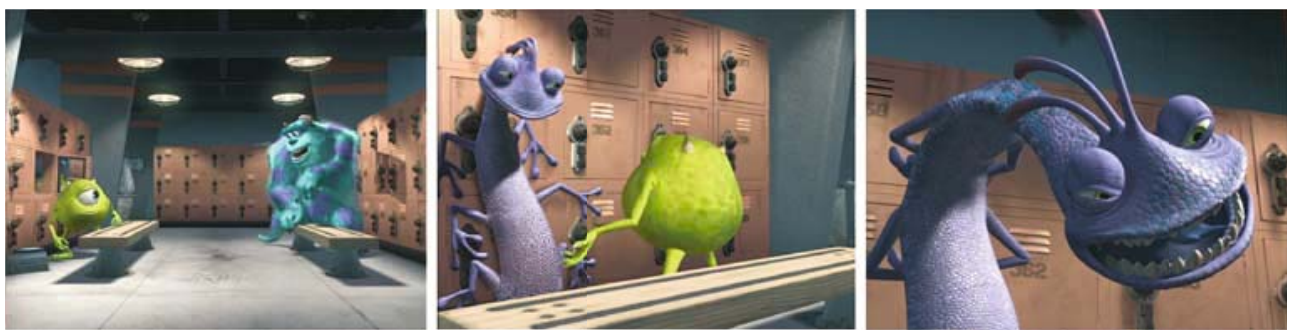
Figures 4-9.
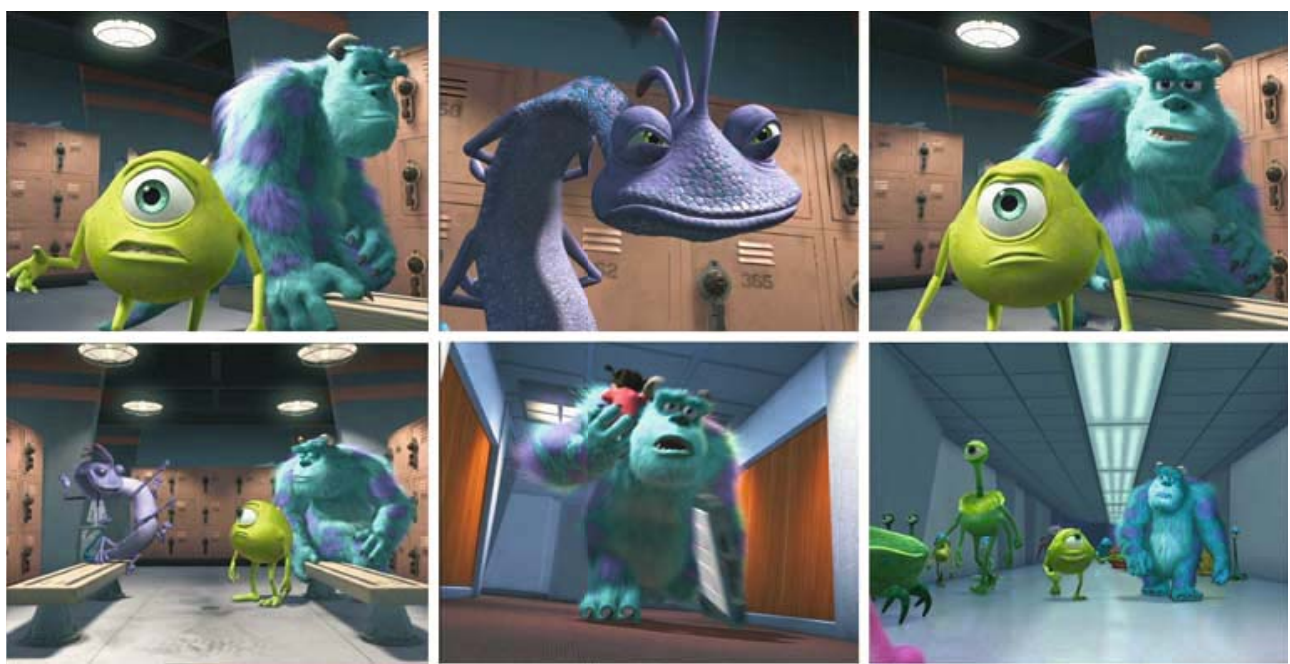

Figures 10-15.
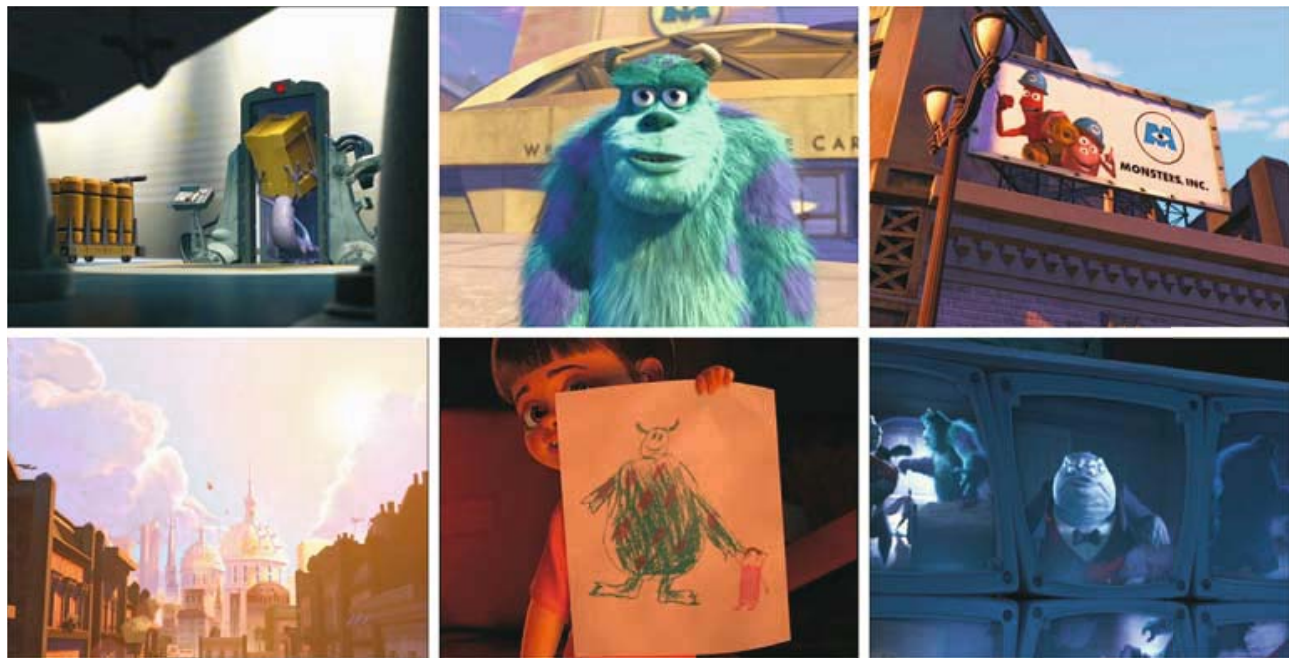